\title{
Atenção à saúde das pessoas com hanseníase: atuação do enfermeiro em unidades básicas de saúde
}

\author{
Health care for people with leprosy: the role of nurses in basic health units
}

Atención a la salud de las personas con lepra: el papel del enfermero en las unidades básicas de salud

Karla Pires Moura Barbosa ${ }^{1 *}$, Tahisa Ferreira da Silva ${ }^{2}$, Eliane Maria Ribeiro de Vasconcelos ${ }^{1}$, Maria Ilk Nunes de Albuquerque ${ }^{1}$, Marta Maria Francisco¹, Yuri Matheus Braga Ferreira Figueiredo.

\section{RESUMO}

Objetivo: Analisar a prática de enfermeiros relacionada às ações de controle da hanseníase nas Unidades de Saúde da Família de um município de Pernambuco. Métodos: Trata-se de um estudo descritivo, com abordagem quantitativa, de corte transversal. A população foi composta por 33 enfermeiros e utilizou-se uma entrevista semiestruturada para a coleta de dados. Os dados foram processados no programa EPIINFO, exportado para o SPSS. O estudo foi aprovado por Comitê de Ética em Pesquisa. Resultados: Os enfermeiros conhecem e operacionalizam as ações de controle da hanseníase, no entanto, apresentam dificuldades na operacionalização de algumas das ações e em suas atribuições no atendimento às pessoas com a doença. Associações estatísticas foram realizadas para identificar os fatores que influenciam no conhecimento, na presença de dificuldades e operacionalização das ações de controle da hanseníase, demonstrando que o conhecimento acerca das ações intervém diretamente na sua realização. Conclusão: Assim, para que os indicadores epidemiológicos revelem taxas menores de detecção e de prevalência de hanseníase faz-se necessário a realização efetiva das ações de controle da hanseníase e a falta de operacionalização dessas ações é consequência da ausência de uma política de educação permanente para os profissionais de saúde.

Palavras-chave: Enfermagem, Hanseníase, Estratégia saúde da família, Promoção da saúde.

\begin{abstract}
Objective: To analyze the practice of nurses related to leprosy control actions in Family Health Units in a municipality in Pernambuco. Methods: This is a descriptive study, with a quantitative, cross-sectional approach. The population was composed of 33 nurses and a semi-structured interview was used for data collection. The data were processed in the EPIINFO program, exported to SPSS. The study was approved by the Research Ethics Committee. Results: The interviewees are familiar with and operationalize the leprosy control actions. However, they have difficulties in operationalizing some of the actions and in their attributions in assisting people with the disease. Statistical associations were made to identify the factors that influence knowledge, in the presence of difficulties and the operationalization of leprosy control actions, demonstrating that knowledge about the actions directly intervenes in the realization of them. Conclusion: Thus, for the epidemiological indicators to reveal lower rates of leprosy detection and prevalence it is necessary to carry out leprosy control actions effectively and the lack of operationalization of these actions is a consequence of the absence of a permanent education policy for health professionals.
\end{abstract}

Keywords: Nursing, Leprosy, Family health strategy, Health promotion.

${ }_{1}^{1}$ Universidade Federal de Pernamuco, Recife - PE. *E-mail: karlapires.mb@gmail.com

2 Instituto de Medicina Integral Professor Fernando Figueira, Recife - PE. 


\section{RESUMEN}

Objetivo: Analizar la práctica de las enfermeras relacionadas con las acciones de control de la lepra en las Unidades de Salud de la Familia de un municipio de Pernambuco. Métodos: Se trata de un estudio descriptivo, con enfoque cuantitativo y transversal. La población estuvo compuesta por 33 enfermeras y se utilizó una entrevista semiestructurada para la recolección de datos. Los datos fueron procesados en el programa EPIINFO, exportados a SPSS. El estudio fue aprobado por el Comité de Ética en Investigación. Resultados: Los entrevistados conocen y operativizan las acciones de control de la lepra. Sin embargo, tienen dificultades para hacer operativas algunas de las acciones y en sus atribuciones para ayudar a las personas con la enfermedad. Se realizaron asociaciones estadísticas para identificar los factores que influyen en el conocimiento, ante la presencia de dificultades y la operacionalización de las acciones de control de la lepra, demostrando que el conocimiento sobre las acciones interviene directamente en la realización de las mismas. Conclusión: Así, para que los indicadores epidemiológicos revelen menores tasas de detección y prevalencia de lepra, es necesario realizar acciones de control de la lepra de manera efectiva y la falta de operatividad de estas acciones es consecuencia de la ausencia de una política de educación permanente para los profesionales de la salud.

Palabras clave: Enfermería, Lepra, Estrategia de salud familiar, Promoción de la salud.

\section{INTRODUÇÃO}

Hanseníase é uma doença crônica, infectocontagiosa, causada pelo Mycobacterium leprae. Apresenta maior prevalência em países subdesenvolvidos, nos quais as condições ambientais são precárias e há grandes desigualdades sociais, o que caracteriza a hanseníase como doença negligenciada. A sintomatologia afeta a pele e pode acometer o sistema nervoso periférico. A transmissão ocorre de uma pessoa infectada, sem tratamento, que libera bacilos por meio das vias aéreas superiores (mucosa nasal e orofaringe), para contatos de convivência íntima e prolongada, frequentemente, intradomiciliares (SEGURADO AC, et al., 2016).

Os principais sinais e sintomas são manchas na pele de coloração esbranquiçada, parda ou eritematosa, em qualquer parte do corpo, com alteração da sensibilidade térmica, tátil e dolorosa; bem como a perda dos pelos e da transpiração no local. Poderá afetar, além da pele, os nervos periféricos que, quando lesionados podem resultar em dormência e perda do tônus muscular. É uma doença curável e o tratamento é realizado com fármacos de administração oral. Os esquemas terapêuticos são específicos, padronizados pelo Ministério da Saúde, denominados como poliquimioterapia (PQT), disponibilizados, exclusivamente, pelos serviços de saúde do SUS. Associados ao tratamento, com a PQT, e na perspectiva de erradicar a doença faz-se necessário a realização de atividades de educação em saúde, para a população de forma sistemática, preferencialmente, por meio de metodologias ativas as quais permitem maior interação entre os sujeitos (SOUZA LR, et al., 2019).

De acordo com o Sistema de Informação de Agravos de Notificação (SINAN), da Secretaria de Vigilância em Saúde (SVS), do Ministério da Saúde (MS), no ano de 2017, Pernambuco apresentou, para a população geral e em menores de 15 anos, uma taxa de detecção de hanseníase de 25,44 casos e 8,60 casos/100 mil habitantes, respectivamente, sendo sua situação caracterizada como endemicidade muito alta, segundo parâmetros do MS, para ambos os casos (BRASIL, 2017; BRASIL, 2016a).

No Brasil, Pernambuco ocupa o $8^{\circ}$ lugar em coeficiente de detecção geral e $7^{\circ}$ em detecção em menores de 15 anos. Na região Nordeste, Pernambuco ocupa o $3^{\circ}$ lugar na detecção geral e em menores de 15 anos. A elevada taxa de detecção, em menores de 15 anos, recomenda prioridade nas ações de controle da hanseníase, que revelem focos de infecção ativos e, com transmissão recente, provavelmente intradomiciliares (BRASIL, 2017; BRASIL, 2016a).

Devido às elevadas taxas de detecção da hanseníase, em Pernambuco, e da concentração de casos na Região Metropolitana do Recife (RMR), que demonstram um importante problema de saúde pública, este estudo objetiva analisar a prática de enfermeiros relacionadas às ações de controle da hanseníase nas Unidades de Saúde da Família de um munícipio de Pernambuco. 


\section{MÉTODOS}

Trata-se de um estudo descritivo de corte transversal com abordagem quantitativa, como recorte de uma pesquisa intitulada "Atuação dos trabalhadores de saúde: médico e enfermeiro da estratégia de saúde da família no programa de controle da hanseníase", a qual estava vinculada ao Programa Nacional de Cooperação Acadêmica (PROCAD), que financiou o estudo e bolsa de Iniciação Científica a duas estudantes do curso de enfermagem.

A coleta de dados foi realizada em um munícipio do estado de Pernambuco, que abrange 12 bairros, e foi realizada no segundo semestre de 2017.

A população era composta por 40 enfermeiros que atuavam nas unidades de saúde da família (USF). Contudo, a amostra final constituiu-se por 33 enfermeiros que atenderam aos critérios de inclusão. Os critérios de exclusão foram: o tempo de atuação na estratégia de saúde da família inferior há um ano e o afastamento por férias, licenças, entre outros.

Utilizou-se para a coleta de dados a entrevista semiestruturada, construída com base nas "Diretrizes para vigilância, atenção e eliminação da hanseníase como problema de saúde pública: manual técnicooperacional" (BRASIL, 2016b). Para maior fidedignidade, aplicou-se um pré-teste com o instrumento proposto, buscando a correção de possíveis erros e a validação.

O banco de dados foi processado no programa EPIINFO, versão 3.5.2 e, exportado para o SPSS, versão 18. Obtiveram-se frequências absolutas e relativas, das variáveis contidas no instrumento, as quais foram submetidas ao teste de significância, através do cálculo do p-valor, e apresentadas na forma de gráficos e de tabelas. A análise dos resultados baseou-se no referencial da literatura, de relevância e pertinência ao tema, nos últimos 10 anos.

A coleta de dados iniciou-se após a aprovação do Comitê de Ética em Pesquisa (CEP) do CCS/UFPE, sob o parecer consubstanciado de № 2.206.374, conforme a Resolução 466/2012 do Conselho Nacional de Saúde (CNS). As entrevistas foram agendadas de acordo com a disponibilidade dos profissionais e realizadas após assinatura do Termo de Consentimento Livre e Esclarecido (TCLE).

\section{RESULTADOS}

$\mathrm{Na}$ análise, observou-se que o tempo mínimo de trabalho dos enfermeiros, na USF de atuação, variou de um ano a 17 anos, em média, 9,5 anos, com desvio padrão de 4,2. Em relação ao tempo de atuação na atenção básica, obteve-se o tempo mínimo de 8 anos e de 28 anos no máximo, perfazendo uma média de 14,7 anos com desvio padrão de 5,3.

Todos os participantes referiram a realização de capacitações sobre diversos temas que envolveram o processo de trabalho em relação à hanseníase. O número máximo de capacitações foi de cinco eventos, por profissional, com média de 2,6 e desvio padrão de 1,3. Porém, os enfermeiros ressaltaram que, na atual gestão municipal, não houve capacitações de relevância sobre a temática. Isso foi elencado como uma das dificuldades para o atendimento de pessoas com hanseníase.

A maioria dos entrevistados, 90,9\%, já havia atendido pessoas com hanseníase, na unidade de saúde. Contudo, no momento, relataram que não havia casos da doença sendo acompanhados nas unidades. Entre os casos detectados, há algum tempo (com variações de intervalos temporais entre os entrevistados), 66,7\% ocorreram por meio do atendimento por demanda espontânea e 30,3\% por busca ativa.

$\mathrm{Na}$ tabela a seguir verificam-se as questões relacionadas ao conhecimento e a operacionalização das ações de controle da hanseníase. A maior frequência, quanto ao conhecimento, está relacionada às ações de vigilância epidemiológica, exame de contatos, orientação e administração da vacina BCG. $\mathrm{Na}$ operacionalização, a ação de maior frequência foi a de educação em saúde. A ação menos conhecida e, consequentemente, realizada foi a prevenção e o tratamento de incapacidades (Tabela 1). 
Tabela 1 - Conhecimento e operacionalização das ações de controle da hanseníase em um munícipio de Pernamuco - PE, 2017.

\begin{tabular}{|c|c|c|c|c|c|c|c|c|}
\hline \multirow{3}{*}{ Ações de Controle } & \multicolumn{4}{|c|}{ Conhecimento referido } & \multicolumn{4}{|c|}{ Operacionalização } \\
\hline & \multicolumn{2}{|c|}{ Sim } & \multicolumn{2}{|c|}{ Não } & \multicolumn{2}{|c|}{ Sim } & \multicolumn{2}{|c|}{ Não } \\
\hline & $\mathrm{N}$ & $\%$ & $\mathrm{~N}$ & $\%$ & $\mathrm{~N}$ & $\%$ & N & $\%$ \\
\hline Educação em saúde & 31 & 93,9 & 2 & 6,1 & 31 & 93,9 & 2 & 6,1 \\
\hline $\begin{array}{c}\text { Investigação } \\
\text { epidemiológica para } \\
\text { o diagnóstico } \\
\text { oportuno de casos }\end{array}$ & 31 & 93,9 & 2 & 6,1 & 2 & 87,9 & 4 & 12,1 \\
\hline $\begin{array}{c}\text { Tratamento até a } \\
\text { cura }\end{array}$ & 30 & 90,9 & 3 & 9,1 & 27 & 81,8 & 6 & 18,2 \\
\hline $\begin{array}{c}\text { Prevenção e } \\
\text { tratamento de } \\
\text { incapacidades }\end{array}$ & 25 & 75,8 & 8 & 24,2 & 20 & 60,9 & 13 & 39,4 \\
\hline $\begin{array}{c}\text { Vigilância } \\
\text { epidemiológica }\end{array}$ & 32 & 97,0 & 1 & 3,0 & 28 & 84,8 & 5 & 15,2 \\
\hline $\begin{array}{l}\text { Exames de contato, } \\
\text { orientação e } \\
\text { aplicação de BCG }\end{array}$ & 32 & 97,0 & 1 & 3,0 & 29 & 87,9 & 3 & 9,1 \\
\hline
\end{tabular}

Fonte: Barbosa KPM, et al., 2017.

Indagados sobre as dificuldades relacionadas à execução das ações de controle, 55,2\% dos enfermeiros destacaram o exame de contatos, orientação e aplicação de BCG, seguidas da prevenção e do tratamento de incapacidades com $55 \%$ e educação em saúde com $45,2 \%$.

Em relação à educação em saúde e a prevenção e tratamento de incapacidades, os maiores obstáculos, com $15,2 \%$, estavam relacionados a falta de insumos e ao envolvimento da população, a referência do paciente para serviços especializados e a insegurança para tratamento e avaliação de incapacidades, respectivamente. Os obstáculos quanto ao exame de contatos, orientação e aplicação da vacina BCG estavam associados a restrição no fornecimento da vacina para as unidades, a recusa dos contatos em comparecer a unidade de saúde, bem como a não localização dos mesmos, $21,2 \%$, e a falta de adesão do paciente ao tratamento com $12,1 \%$.

Quanto às atribuições específicas do enfermeiro no controle e prevenção da hanseníase, as que apresentaram maiores frequências foram: consultas de enfermagem; tratamento imediato e orientação dos ACS para o acompanhamento dos casos em tratamento e a programação para dispensação dos fármacos, segundo esquema terapêutico. As que apresentaram menor frequência: avaliação e registro do grau de incapacidade física; prescrição de outras medicações (fora do esquema terapêutico padronizado), conforme protocolo; assistência domiciliar; realização de curativos; avaliação e registro do grau de incapacidade física no momento da alta por cura; orientação e/ou realização de técnica de prevenção de incapacidades físicas; análise de dados para o planejamento de intervenções e o envio mensal das informações epidemiológicas (Tabela 2). 
Tabela 2 - Atribuições dos Enfermeiros no atendimento a casos de hanseníase em um município de Pernambuco -PE, 2017.

\begin{tabular}{|c|c|c|}
\hline Atribuições do enfermeiro & $\mathbf{n}$ & $\%$ \\
\hline Realiza consulta de enfermagem; & 31 & 93,9 \\
\hline $\begin{array}{c}\text { Identificar sinais e sintomas da hanseníase e avalia os casos suspeitos } \\
\text { encaminhados para a unidade de saúde; }\end{array}$ & 30 & 90,9 \\
\hline Realizar avaliação dermatoneurológica dos casos suspeitos; & 25 & 75,8 \\
\hline Solicitar exames complementares; & 20 & 60,6 \\
\hline $\begin{array}{l}\text { Preencher ficha individual de notificação para os casos confirmados de } \\
\text { hanseníase; }\end{array}$ & 28 & 84,8 \\
\hline Avaliar e registrar o grau de incapacidade física; & 9 & 27,3 \\
\hline $\begin{array}{l}\text { Realizar tratamento imediato e adequado, de acordo com esquemas } \\
\text { terapêuticos preconizados pelo Ministério da Saúde (PQT/OMS); }\end{array}$ & 31 & 93,3 \\
\hline Prescrever outras medicações, conforme protocolo; & 11 & 33,3 \\
\hline Realizar exame dermatoneurológico em todos os contatos intradomiciliares; & 28 & 84,8 \\
\hline Realizar assistência domiciliar, quando necessário; & 12 & 36,4 \\
\hline Realizar ou demandar a realização de curativos; & 3 & 9,1 \\
\hline $\begin{array}{c}\text { Registrar, em prontuários do usuário e fichas próprias, o exame } \\
\text { dermatoneurológico, bem como evoluções, avaliações de grau de incapacidade, } \\
\text { administração de dose supervisionada e alta; }\end{array}$ & 26 & 78,8 \\
\hline Avaliar e registrar grau de incapacidade no momento da alta por cura; & 5 & 15,2 \\
\hline Orientar o paciente e a família para a realização de auto-cuidado; & 29 & 87,9 \\
\hline Orientar e/ou realizar técnicas simples de prevenção de incapacidades físicas; & 12 & 36,4 \\
\hline $\begin{array}{l}\text { Observar a tomada da dose supervisionada e orientar acerca de efeitos } \\
\text { adversos dos medicamentos; }\end{array}$ & 26 & 78,8 \\
\hline $\begin{array}{l}\text { Orientar os auxiliares/técnicos de enfermagem, ACS para o acompanhamento } \\
\qquad \text { dos casos em tratamento; }\end{array}$ & 31 & 93,9 \\
\hline Planejar, gerenciar, coordenar e avaliar as ações desenvolvidas pelos ACS; & 22 & 66,7 \\
\hline $\begin{array}{l}\text { Analisar os dados e planejar as intervenções juntamente com a equipe de } \\
\text { saúde; }\end{array}$ & 14 & 42,4 \\
\hline $\begin{array}{l}\text { Contribuir e participar das atividades de educação permanente dos membros } \\
\qquad \text { da equipe; }\end{array}$ & 23 & 69,7 \\
\hline Desenvolver ações educativas e de mobilização; & 23 & 69,8 \\
\hline $\begin{array}{l}\text { Realizar a programação e pedidos de medicamentos e controlar o estoque em } \\
\text { formulário específico e encaminhá-lo ao nível pertinente. }\end{array}$ & 33 & 100,0 \\
\hline $\begin{array}{l}\text { Encaminhar ao setor competente a ficha de notificação e boletins de } \\
\text { acompanhamento, conforme estratégia local; }\end{array}$ & 30 & 90,9 \\
\hline Enviar mensalmente ao setor competente as informações epidemiológicas; & 15 & 45,6 \\
\hline
\end{tabular}

Fonte: Barbosa KPM, et al., 2017. 
Para o teste de significância, $p$-valor deveria ser $<0,05$ para que houvesse associação. No estudo houve significância na associação do conhecimento das ações de controle quando associadas à realização e no conhecimento sobre a dificuldade na realização das mesmas, nas seguintes variáveis: investigação epidemiológica para o diagnóstico oportuno de casos ( $p$-valor $=0,011)$, tratamento até a cura $(p$-valor $=0,004)$ e prevenção e tratamento de incapacidades ( $p$-valor $=0,035)$.

\section{DISCUSSÃO}

O estudo demonstrou que os entrevistados conhecem, operacionalizam e identificam os obstáculos quanto a realização das ações de controle da hanseníase e as atribuições específicas para o atendimento dos casos.

Os enfermeiros destacaram que, dentre as capacitações realizadas, algumas foram relacionadas ao controle da hanseníase, porém não souberam precisar a quantidade. Enfatizaram, que na gestão atual, não houve capacitações sobre o tema, acarretando a insegurança para executarem ações de controle. Resultados semelhantes aos obtidos no estudo de Rodrigues FF, et al. (2015), realizado com enfermeiros da atenção primária à saúde, que demonstrou que as capacitações influenciam na assistência prestada. A educação permanente é um instrumento importante para a conscientização dos profissionais sobre a hanseníase, enquanto problema de saúde pública, sendo indispensável para a atuação profissional. É uma forma de melhorar o conhecimento dos profissionais, diante da insegurança para o desenvolvimento das ações específicas.

O presente estudo demonstra que a maioria, $90,9 \%$, dos enfermeiros já atenderam casos de hanseníase, no entanto, no momento da pesquisa, não havia pessoas em tratamento nas USF. O que é contraditório com a situação epidemiológica do município de Recife, apresentada nos indicadores. Esse fato indica que há fragilidades de detecção dos casos novos, na captação precoce e, consequentemente, ocorre uma limitação em assumir a responsabilidade pelo diagnóstico presuntivo.

Os fatos acarretados pela situação epidemiológica contraditória do município acabam culminando no aumento do número de casos, também, em menores de 15 anos. Dado que sinaliza um déficit na realização das ações de controle e um agente disseminador ativo, pois, quando há uma criança com hanseníase, possivelmente, existe um adulto com hanseníase em seu convívio intradomiciliar. Além disso, grande parte dos casos detectados, neste estudo, foi por demanda espontânea e uma pequena parcela por busca ativa, o que ratifica a falta de captação precoce dos casos.

Dados que corroboram com os que foram encontrados nos estudos de Lima RSK, et al. (2016), Neta OAG, et al. (2017), Lana FCF, et al. (2007, 2008), em que a busca ativa, mesmo sendo uma ação preconizada pelo Ministério da Saúde como uma forma eficaz de diagnosticar e tratar precocemente os casos, é pouco realizada pelos profissionais de saúde, onde a maioria são notificados de forma passiva, quando o paciente procura o serviço de saúde. Essa possível passividade dos serviços de saúde contribui para o aparecimento de novos casos e por serem diagnosticadas tardiamente, geram incapacidades físicas nos indivíduos acometidos pela doença.

A pesquisa demonstra que as ações de vigilância epidemiológica, o exame de contato, a orientação e aplicação da vacina BCG foram as mais conhecidas. Quanto à operacionalização, a Educação em Saúde obteve destaque. As ações de prevenção e o tratamento de incapacidades foram as que apresentaram a menor taxa de identificação e realização, enquanto ação de controle, consequência do déficit no conhecimento por parte dos profissionais, referindo que se há uma incapacidade presente, não houve um controle da doença. Diante disso, os profissionais por insegurança optam por referenciar a pessoa doente para um serviço especializado.

Segundo Nascimento GRC, et al. (2011), os enfermeiros entendem como vigilância epidemiológica a busca ativa realizada pelos ACS e controle dos contatos. De certa forma é uma ação facilitada pelos ACS, quando realizam a visita domiciliar, mas seu objetivo é mais amplo e é efetuado pelo conjunto de ações que gera dados sobre a doença e o seu perfil epidemiológico, que indica, efetua e avalia as ações de controle, e também fornece informação para os profissionais e a população. 
Estudo realizado por Souza MF, et al. (2017) em Camaragibe, município do estado de Pernambuco que pertence à região metropolitana de Recife, indica que, dentre as várias causas, as ações de vigilância epidemiológica precárias cooperam no aumento da taxa de detecção geral da doença e em sua endemicidade. Um número crescente de casos em menores de 15 anos, além de configurar uma região como hiperendêmica, revela a continuidade da transmissão e a exposição precoce a focos ativos, propondo déficit na vigilância e no controle da hanseníase (PIRES CAA, et al., 2012).

De acordo com Crispim JA, et al. (2013) e Silva PLN (2012), a educação em saúde é uma atividade importante, cujo objetivo é informar quanto a prevenção de doenças e de agravos e a promoção da saúde. Uma ação que pode transformar o comportamento individual e coletivo, promovendo uma melhor qualidade de vida e saúde (MALLMANN DG, et al., 2015).

A ação educacional deve ser planejada segundo as características sociais, econômicas e culturais de cada grupo, para que a mesma esteja ao alcance da população, podendo utilizar as tecnologias educacionais para auxiliar na transmissão da informação, aspirando que a população alvo se aproprie desse conhecimento (LOPES EFB, et al., 2020; SILVA DML, et al., 2017).

Nessa perspectiva, a equipe de enfermagem pode utilizá-la para estabelecimento do cuidado em saúde dos indivíduos e da comunidade, transmitindo o conhecimento como um modo de prevenir e melhorar a saúde e a qualidade de vida de todos. O desenvolvimento de ações de educação, relacionadas a hanseníase, é fundamental para prevenção da doença, promoção de saúde, adesão ao tratamento e combate ao estigma da doença (LOPES EFB, et al., 2020).

Em relação à prevenção e ao tratamento de incapacidades, o resultado do estudo se aproximou ao de Pinhiero JJG, et al. (2017), no qual refere que muitos profissionais de saúde não apresentam conhecimento sobre as técnicas de avaliação e classificação do grau de incapacidades, demonstrando que a falta de capacitação dos profissionais de saúde, com relação à essa ação de controle, tem como consequência a não realização e insegurança para o diagnóstico da doença. Uma vez que os profissionais são capacitados, eles sentem-se seguros para avaliar os pacientes, prevenindo e/ou tratando as incapacidades.

Ainda em relação à prevenção e tratamento de incapacidades, existe a falta de sua relevância como ação de controle, situação evidenciada pela baixa frequência de sua citação em trabalhos científicos acerca das ações de controle da hanseníase (CASTRO C e VERAS RL, 2019) e pela falta de sua continuidade, por parte daqueles que a operacionalizam, visto que, a mesma é feita em menor proporção após a cura quando comparada ao momento do diagnóstico (ALVES ES, et al., 2017). A prevenção e o tratamento de incapacidades precisam estar associadas às demais ações de controle, pois, a mesma, além de contribuir para a vigilância epidemiológica, é empregada como indicador de controle da doença (AJALLA MEA, et al., 2016; ALVES ES, et al., 2017).

As atribuições específicas do enfermeiro, no controle e prevenção da hanseníase, apresentadas no estudo através da Tabela 2, devem ser realizadas, preferencialmente, nas unidades de saúde após a confirmação dos casos. Após o diagnóstico, o atendimento pelo enfermeiro deverá ser feito de forma integral, informar e esclarecer as dúvidas acerca da doença, oferecer apoio durante o tratamento, orientar o indivíduo sobre o autocuidado e a prevenção de incapacidades. As ações de enfermagem são importantes para o controle da hanseníase, assim, as mesmas devem ser realizadas de forma efetiva (NASCIMENTO GRC, et al., 2011; AGUIAR PG, et al., 2014).

Para a consulta de enfermagem é fundamental o profissional capacitado, principalmente na avaliação do exame dermatoneurológico, prevenção e tratamento de incapacidades e na assistência a pessoas que apresentarem reações hansênicas, atribuições que apresentam menor realização devido à falta de segurança por parte dos profissionais. O diálogo entre profissional e pessoa com hanseníase sobre todos os aspectos da patologia é importante para o controle da doença, adesão a PQT e o incentivo ao autocuidado que previne incapacidades (AGUIAR PG, et al., 2014; RAMOS JS, et al., 2019).

Chama atenção a baixa frequência das visitas domiciliares, por parte dos enfermeiros. Ação que, além de oportunizar a vigilância em saúde e acompanhar a população (VIEIRA NF, et al., 2018), aprimora a busca 
ativa de pacientes sintomáticos e assintomáticos, adesão e continuidade do tratamento. Quando somada às demais, e implementadas, os resultados deverão ser monitorados e avaliadas para melhorar a eficácia no controle da hanseníase. É indispensável à participação de todos os profissionais da equipe de saúde, para a interrupção da cadeia de transmissão, com identificação da fonte da disseminação e detecção de novos casos. Nesse contexto, o enfermeiro tem como uma das atribuições mais importantes capacitar os profissionais, que estão sob sua responsabilidade, o que possibilita a ampliação do cuidado (NASCIMENTO GRC, et al., 2011)

Na correlação entre o conhecimento e as dificuldades na realização das ações com o tempo de trabalho, obteve-se $p$-valor $>0,05$, significando que o tempo de trabalho não é capaz de influenciar no conhecimento ou presença de dificuldades na realização das ações de controle. Quanto à associação do número de capacitações com o conhecimento das ações foi obtido $p$-valor $>0,05$, que afirma que o número de capacitações não foi capaz de influenciar no conhecimento das ações de controle. A relação do conhecimento sobre as ações de controle e a realização e dificuldades na operacionalização foi a única associação que teve significância, pois obteve $p$-valor $<0,05$ nas seguintes ações de controle: a investigação epidemiológica para o diagnóstico oportuno de casos, o tratamento até a cura e a prevenção de tratamento de incapacidades, nas quais, o conhecimento influenciou na realização das mesmas.

As dificuldades encontradas para a coleta de dados, deste estudo, relacionaram-se aos agendamentos das entrevistas, devido ao grande número de atribuições dos profissionais e, consequentemente, da falta de tempo. Recomenda-se a continuidade do estudo para avaliação posterior em outros distritos do município do Recife, no intuito de verificar a conduta dos enfermeiros no atendimento dos casos de hanseníase e na operacionalização das ações de controle, comparando os resultados obtidos, para proposição de alterações.

\section{CONCLUSÃO}

Se não há busca ativa e nem prioridade quanto à realização das ações de controle da hanseníase, há uma redução na detecção e, consequentemente, subnotificação dos casos. Isso significa que os indicadores de hanseníase, no município estudado, podem ocupar parâmetros mais elevados, com indicadores de tendência crescente, mesmo sem casos notificados nas USFs. Acredita-se que as notificações estão ocorrendo nas unidades de referência. Para que esses indicadores sejam revertidos, com redução das taxas de detecção e de prevalência de hanseníase, faz-se necessário a realização efetiva das ações de controle. A dificuldade de operacionalização das ações tem como uma das consequências a ausência de uma política de educação permanente para os profissionais de saúde. O estudo ratifica a hanseníase enquanto uma doença negligenciada, pois há baixo investimento e prioridade nas ações de controle da doença.

\section{AGRADECIMENTOS E FINANCIAMENTO}

Agradecemos ao Programa Nacional de Cooperação Acadêmica (PROCAD), financiado pela Coordenação de Aperfeiçoamento de Pessoal de Nível Superior (CAPES) com parceria entre EERPUSP/UFPE/UEPA/UFAM, sob a coordenação da Professora Dra. Claudia Benedita dos Santos (EERP-USP), em nos disponibilizar duas bolsas de iniciação científica para a realização dessa pesquisa.

\section{REFERÊNCIAS}

1. AGUIAR PG, et al. Fatores de manutenção da endemia hansênica e as ações da enfermagem no controle da hanseníase. Revista de Iniciação Científica da Libertas, 2014; 4: 119-132.

2. AJALLA MEA, et al. The context of leprosy in Brazil-Paraguay. Ciênc saúde coletiva. 2016; 21(1).

3. ALVES ES, et al. Perfil epidemiológico da hanseníase em um município do nordeste brasileiro: uma análise retrospectiva. Revista de Pesquisa Cuidado é Fundamental Online, 2017; 9: 648-652.

4. BRASIL. Ministério da Saúde. Taxa de detecção de hanseníase geral e em menores de 15 anos por 100.000 habitantes, Estados e Regiões, Brasil, 1994-2017. Sinan/SVS - MS, 2017. Disponível em: https://portalarquivos2.saude.gov.br/images/pdf/2018/julho/13/Taxa-de-detecao-dos-casos-novos-de-hanseniaseem-menores-de-15-anos-1994a2017.pdf. Acessado em: 20 de junho de 2018. 
5. BRASIL. Ministério da Saúde. Guia de Vigilância em Saúde. 2016. Disponível em: https://portalarquivos2.saude.gov.br/images/pdf/2016/agosto/25/GVS-online.pdf. Acessado em: 10 de fevereiro de 2017.

6. BRASIL. Ministério da Saúde.Diretrizes para vigilância, atenção e eliminação da Hanseníase como problema de saúde pública: manual técnico-operacional. Brasília: Ministério da Saúde, 2016b. 58p.:il. Disponível em: https://portalarquivos2.saude.gov.br/images/pdf/2016/fevereiro/04/diretrizes-eliminacao-hanseniase-4fev16-web.pdf. Acessado em: 10 de fevereiro de 2017.

7. CASTRO C, VERAS RL. O papel da enfermagem na prevenção de incapacidades físicas em hanseníase: uma revisão bibliográfica. Trabalho de Conclusão de Curso de Graduação de Enfermagem. Centro Universitário São Lucas. Porto Alegre, p. 19. 2019.

8. CRISPIM JA, et al. Tuberculose no contexto das famílias: as vivências de familiares e pacientes acometidos pela doença. Rev enferm UERJ. 2013; 21: 606-11.

9. LANA FCF, et al. Hanseníase em menores de 15 anos no Vale do Jequitinhonha, Minas Gerais, Brasil. Revista Brasileira de Enfermagem, 2007; 60: 696-700.

10. LANA FCF, et al. Desenvolvimento de incapacidades físicas decorrentes da hanseníase no Vale do Jequitinhonha, MG. Revista Latino-americana de Enfermagem, 2008; 16: 993-997.

11. LIMA RSK, et al. A importância da busca ativa como estratégia de controle da hanseníase em territórios endêmicos. Hansen Int. 2016; 41: 55-63.

12. LOPES EFB, et al. Educação em saúde: uma troca de saberes no combate ao estigma da hanseníase. Braz. J. of Develop., 2020; 2: 5350-5368.

13. MALLMANN DG, et al. Educação em saúde como principal alternativa para promover a saúde do idoso. Ciência \& Saúde Coletiva, 2015; 20: 1763-1772.

14. NETA OAG, et al. Percepção dos profissionais de saúde e gestores sobre a atenção em hanseníase na estratégia saúde da família. Revista Brasileira em Promoção da Saúde, 2017; 30: 239-248.

15. NASCIMENTO GRC, et al. Ações do enfermeiro no controle da hanseníase. Revista Eletrônica de Enfermagem, $2011 ; 13: 743-50$

16. PINHEIRO JJG, et al. Aptidões cognitivas e atitudinais do enfermeiro da atenção básica no controle da hanseníase. Rev. baiana enferm, 2017; 31: e17257.

17. PIRES CAA, et al. Leprosy in children under 15 years: the importance of early diagnosis. Rev Paul Pediatr 2012; 30(2): 292-5.

18. RAMOS JS, et al. Dificuldades da enfermagem no manejo da hanseníase na atenção primária. Revista JRG de Estudos Acadêmicos, 2019; 2(5).

19. RODRIGUES FF, et al. Knowledge and practice of the nurse about leprosy: actions of control and elimination. Rev Bras Enferm, $2015 ; 68(2):$ 297-304.

20. SEGURADO AC, et al. Saúde nas metrópoles - Doenças infecciosas. Estud. av. [online], 2016; 30: 29-49.

21. SILVA PLN. Perfil de conhecimentos sobre hanseníase entre moradores de uma Estratégia Saúde da Família. Hansen Int, 2012; 37(2): 31-39.

22. SILVA DML, et al. Tecnologias educacionais na assistência de enfermagem em educação em saúde: revisão integrativa. Rev enferm UFPE on line, 2017; 11(Supl.2): 1044-51.

23. SOBRINHO RAS, et al. Avaliação do grau de incapacidades em hanseníase: uma estratégia para sensibilização e capacitação da equipe de enfermagem. Rev Latino Americana de Enfermagem, 2007.

24. SOUZA LR, et al. Hanseníase: Diagnóstico e tratamento. HUMANIDADES \& TECNOLOGIA EM REVISTA (FINOM), 2019; 16: 1809-1628.

25. SOUZA MF, et al. Avaliação da implantação do programa de controle da hanseníase em Camaragibe - Pernambuco. Epidemiol. Serv. Saude, 2017; 26(4): 817-834.

26. VIEIRA NF, et al. Avaliação dos atributos da atenção primária à saúde nas ações de controle da hanseníase. Rev enferm UERJ, 2018; 26: e31925. 